\section{EXPRESSION OF GALECTIN-3 INHIBITORS FROM A SELF- REPLICATING RNA VECTOR AS TREATMENT FOR PEDIATRIC OSTEASARCOMA}

Guillermo Herrador Cañete*, Marta Zalacain, Sara Labiano, Javier Martinez, Cristian Smerdou, Marta Alonso. Cima Universidad de Navarra, Pamplona, Spain

Background Osteosarcoma is an aggressive bone tumor, primarily arising in the pediatric age. Despite years of intensive research, outcome for metastatic and non-responder patients is very poor and has not improved in the last 30 years. These tumors harbor a highly immunosuppressive environment, making the existing immunotherapies ineffective. Inhibition of galectin-3 (Gal3), a protein involved in immunosuppression, ${ }^{1-3}$ adhesion of tumor cells, ${ }^{4} 5$ and metastases, ${ }^{6-9}$ has been demonstrated to reduce tumor progression in different tumor models, including osteosarcoma. ${ }^{10-12}$ On the other hand, virotherapy based on recombinant Semliki Forest Virus (SFV), a self-replicating RNA virus, has shown therapeutic effect in orthotopic osteosarcoma mouse models. ${ }^{13}$

Methods We generated SFV vectors expressing truncated forms of Gal3, including its carboxy-terminal domain (SFV-Gal3-C) and its amino-terminal domain alone (SFV-Gal3-N) or fused to the Gal3 inhibitor peptide C12 (SFV-Gal3-N-C12). An additional construct expressed the C12 peptide ${ }^{14}{ }^{15}$ (SFV$\mathrm{C} 12)$. We analyzed Gal3 expression in different murine and human osteosarcoma cell lines. Orthotopic osteosarcoma tumors, induced by intratibial injection of K7M2 murine cells, which showed high expression of Gal3, were treated with SFV vectors expressing Gal3 inhibitors or luciferase or with PBS (control). Animals were maintained under standard conditions, and all procedures were approved by the Institutional Ethical Committee (CEEA) in accordance with the guidelines of the University of Navarra, approval number 044-21.

Results Treatment with the SFV-Gal3-N-C12 vector showed the highest antitumor activity, significantly reducing tumor growth compared to control mice that received PBS. In fact, this vector prolonged animal survival, leading to $47 \%$ of complete regressions. Among the other vectors, SFV-Gal3-N and SFV-C12 were also able to transiently decrease tumor growth, although they had no impact on animal survival. Moreover, the number of spontaneous lung metastasis were reduced in mice treated with SFV vectors expressing Gal3 inhibitors. Preliminary mechanistic studies showed an increase of CD3 cells infiltration in tumors treated with SFV-Gal3-N-C12 and SFVGal3-N vectors. Despite the antitumor effect observed with SFV-Gal3-N-C12, no protection against tumor rechallenge was observed in cured mice, indicating the lack or insufficient memory immune response generation. These data suggested that this therapeutic approach might benefit from combination with other immunodulatory strategies. We are currently characterizing the underpinnings of the mechanisms underlying this strategy.

Conclusions In summary, we believe that inhibition of Gal3 using SFV vectors could constitute a potential approach to explore as therapy for pediatric osteosarcoma.

\section{REFERENCES}

1. Farhad M, Rolig AS, Redmond WL. The role of Galectin-3 in modulating tumor growth and immunosuppression within the tumor microenvironment. Oncoimmunology 2018;7(6):p.e1434467

2. Ruvolo PP. Galectin 3 as a guardian of the tumor microenvironment. Biochim Biophys Acta 2016;1863(3):427-437.

3. Guo $Y$, et al. Roles of galectin3 in the tumor microenvironment and tumor metabolism (Review). Oncol Rep 2020:44(5):1799-1809.
4. Cao $Z$, et al. Endogenous and exogenous galectin-3 promote the adhesion of tumor cells with low expression of MUC1 to HUVECs through upregulation of $\mathrm{N}$ cadherin and CD44. Lab Invest 2018;98(12):1642-1656.

5. Kim SJ, Chun KH. Non-classical role of Galectin-3 in cancer progression: translocation to nucleus by carbohydrate-recognition independent manner. BMB Rep 2020;53(4):173-180.

6. Pereira JX, et al. Galectin-3 regulates the expression of tumor glycosaminoglycans and increases the metastatic potential of breast cancer. J Oncol 2019:2019:9827147.

7. Song $M$, et al. Galectin-3 favours tumour metastasis via the activation of betacatenin signalling in hepatocellular carcinoma. Br J Cancer 2020;123(10):15211534

8. Fortuna-Costa A, et al. Extracellular galectin-3 in tumor progression and metastasis. Front Oncol 2014:4:138.

9. Nakajima K, et al. Galectin-3 in bone tumor microenvironment: a beacon for individual skeletal metastasis management. Cancer Metastasis Rev 2016;35(2):33346.

10. Nakajima K, Balan V, Raz A. Galectin-3: an immune checkpoint target for musculoskeletal tumor patients. Cancer Metastasis Rev 2021:40(1):297-302.

11. Lei $P$, et al. Small interfering RNA-induced silencing of galectin-3 inhibits the malignant phenotypes of osteosarcoma in vitro. Mol Med Rep 2015;12(4):631622.

12. Park $G B$, et al. Silencing of galectin-3 represses osteosarcoma cell migration and invasion through inhibition of FAK/Src/Lyn activation and beta-catenin expression and increases susceptibility to chemotherapeutic agents. Int J Oncol 2015;46 (1):185-94.

13. Ketola $A$, et al. Oncolytic Semliki forest virus vector as a novel candidate against unresectable osteosarcoma. Cancer Res 2008:68(20):8342-50.

14. Sun W, et al. G3-C12 peptide reverses galectin-3 from Foe to friend for active targeting cancer treatment. Mol Pharm 2015;12(11):4124-36.

15. Sun W, et al. Two birds, one stone: dual targeting of the cancer cell surface and subcellular mitochondria by the galectin-3-binding peptide G3-C12. Acta Pharmacol Sin 2017:38(6):806-822.

Ethics Approval Animals were maintained under standard conditions, and all procedures were approved by the Institutional Ethical Committee (CEEA) in accordance with the guidelines of the University of Navarra, approval number 044-21.

http://dx.doi.org/10.1136/jitc-2021-SITC2021.741 